\title{
Synthesis Conditions for LPV Controller with Input Covariance Constraints
}

https://doi.org/10.1515/eng-2019-0018

Received March 4, 2019; accepted May 13, 2019

\begin{abstract}
In this paper, novel synthesis conditions for state-feedback Linear Parameter Varying (LPV) controller with Input Covariance Constraints (ICC) are developed. The synthesis conditions achieve the following design requirements 1) some constraints need to be satisfied on the control energy and 2) optimizing the performance outputs for the entire parameter space of the LPV system. These conditions are formulated as convex optimization problem with Parameterized Linear Matrix Inequalities (PLMIs) constraints. The effectiveness of the proposed approach is illustrated through numerical examples.
\end{abstract}

Keywords: LPV systems, Robust Control, Gain-scheduling, Input Covariance Constraints.

\section{Introduction}

Linear Parameter Varying (LPV) control techniques witnessed great interest in the past three decades [1, 2]. LPV systems can be divided into two main categories, gridding and polytopic LPV models [3]. The gridding LPV control is based on linearizations at specific local points, therefore, there is no guarantee of stability and/or performance for the entire range of the parameter space [4]. On the other hand, polytopic LPV systems provide this guarantee in addition to the simplicity of controller design [5]. Lyapunov approach is used to analyze stability for polytopic LPV systems [6-8].

Since all actuators require limited amount of the available power, practical control systems have to operate below a specified constraints. In the case of designing controllers using classical design methods, the limits of the operation of the actuators are not considered through the

\footnotetext{
*Corresponding Author: Lina Alhmoud: Department of Electrical Engineering,Yarmouk University, Irbid 21163, Jordan, E-mail:lina.hmoud@yu

Ali Khudhair Al-Jiboory: Mechanical Engineering, University of Diyala, Baqubah 32001, Iraq, E-mail: ali_khudhair@uodiyala.edu.iq
}

design stage. Therefore, the controller could command the actuator to operate beyond its allowable range that may damage the actuator. Therefore, it is always required to keep the actuators working in their linear range and satisfying their constraints. If the control signal exceeds the constraints, the closed-loop system will operate beyond its linear range and even stability may no longer guaranteed. This control problem can be solved via Input Covariance Constraint (ICC) controller [9, 10].

The ICC control problem is a problem that optimize output performance such that constraints on the control input are satisfied. In other words, it guarantees the actuators work within their linear range of operation.

The ICC control problem can be interpreted in two ways, deterministic and stochastic interpretation. In the deterministic interpretation, the external disturbance inputs are assumed to belong to a bounded energy $\mathcal{L}_{2}$ set. By defining these inputs this way, the ICC control problem is the problem of minimizing the weighted sum of worst-case peak values on the performance outputs subject to the constraints on the worst-case peak values of the control input. On the other hand, these external inputs can be considered uncorrelated zero-mean white noises with specified intensity. Thus, the ICC control problem minimizes the weighted output performance subject to the control input covariance constraints.

The ICC control problem has been studied earlier for Linear Time Invariant (LTI) systems in [11]. Then, the problem had been revisited in [12] with Linear Matrix Inequalities (LMIs) approach. Tensegrity simplex structure is studied in [13] with ICC controller to satisfy torque actuator constraints. In [14], electric throttle is investigated with experimental validation of ICC controller. The constrained LQ control problem is studied in [15] that minimizes the control energy while satisfying Output Covariance Constraints (OCC).

As far as the author's know, this control problem has never been studied for LPV systems before. Therefore, the objective of this paper is to extend the ICC control problem presented in [12] for Linear Time-Invariant (LTI) to handle LPV systems. More specifically, we have developed new synthesis conditions to design state-feedback LPV controller to asymptotically stabilize the closed-loop sys- 
tems while satisfying constraints on control signals. These synthesis conditions are formulated as convex optimization problem with Parametrized Linear Matrix Inequalities (PLMIs) constraints. Thus, the developed conditions have infinite constraints. Therefore, matrix coefficient check approach has been used to relax these conditions into a finite set of numerically tractable LMIs [16].

The paper is organized as follows. Section 2 presents preliminaries of the LPV systems and signals norm. Section 3 presents the synthesis conditions of the ICC LPV controller. Numerical examples are given in Section 4 to demonstrate the benefit of the developed conditions. Closing conclusions are given in section 5

Standard notation is used in this paper. $\mathbb{R}$ denotes the set of real numbers. The symbol " $T$ " denotes transpose of a matrix. The matrices $\boldsymbol{I}_{n}$ and $\mathbf{0}_{n}$ denote an identity and zero matrices of size $n \times n$, respectively. The positive definiteness of a matrix $A$ is denoted $A>0$. The sum of the diagonal elements of a matrix $A$ is referred as $\operatorname{trace}(A)$. The transposition of the off-diagonal matrix block is referred by " ”. Other notations will be illustrated in due course.

\section{Preliminaries}

Consider the following linear parameter varying system :

$$
\begin{aligned}
& \dot{x}(t)=A(\theta(t)) x(t)+B_{u}(\theta(t)) u(t)+B_{w}(\theta(t)) w(t), \\
& y(t)=C(\theta(t)) x(t) \\
& A(\theta(t))=A_{0}+\sum_{i=1}^{q} \theta_{i}(t) A_{i}, \quad i=1,2, \cdots, q .
\end{aligned}
$$

where $q$ is the number of scheduling parameters. The scheduling parameters are assumed to be bounded such that,

Moreover, the rate of change of the scheduling parameters are assumed to be bounded as well.

The LPV system in (1) can be converted into polytopic coordinate using the change of variables,

$$
\mu_{1}(t)=\frac{\theta(t)-\underline{\theta}}{\bar{\theta}-\underline{\theta}}, \quad \mu_{2}(t)=1-\mu_{1}(t),
$$

where $\mu:=\left(\mu_{1}(t), \mu_{2}(t)\right) \in \Lambda_{2}$ and

$$
\Lambda_{2}=\left\{\mu(t) \in \mathbb{R}^{2}: \sum_{i=1}^{2} \mu_{i}(t)=1, \mu_{i}(t) \geq 0, i=1,2\right\},
$$

where the subscript "2" denotes the number of vertices. Following the procedure presented in $[17,18]$, the derivatives of the varying parameters can assume values bounded by convex polytope $\Omega$.

Thus, the system in (1) can be expressed in the polytopic coordinates (in terms of $\mu(t)$ ) as

$$
\begin{aligned}
& \dot{x}(t)=A(\mu(t)) x(t)+B_{u}(\mu(t)) u(t)+B_{w}(\mu(t)) w(t), \\
& y(t)=C(\mu(t)) x(t) .
\end{aligned}
$$

The $\mathcal{L}_{2}$ definition of a signal is given by

$$
\|w(t)\|_{2}^{2}:=\int_{0}^{\infty} w(t)^{T} w(t) d t
$$

while the $\mathcal{L}_{\infty}$ is defined

$$
\|y(t)\|_{\infty}^{2}:=\sup _{t \geq 0} y(t)^{T} y(t),
$$

then, the $\mathcal{L}_{2}$-to- $\mathcal{L}_{\infty}$ gain for Linear Time Invariant (LTI) systems is defined by

$$
\|T\|_{2}^{2}:=\sup _{t \geq 0} \frac{\|y(t)\|_{\infty}^{2}}{\|w(t)\|_{2}^{2}}
$$

where $T$ is the transfer function from the disturbance input $w(t)$ to performance output $y(t)$.

\section{Input Covariance Constraints (ICC)}

The control objective is to design gain-scheduling statefeedback controllers of the form

$$
u(t)=K(\mu(t)) x(t)
$$

in order to:

- stabilize the closed-loop system.

- satisfy constraints on input covariances defined by $\bar{U}$.

- minimize the output performance in terms of the $\mathcal{H}_{2}$ norm of the closed-loop system from the disturbance input $w(t)$ to $y(t)$.

Augmenting the open-loop system (1) with the controller (8), the following closed-loop system matrices are obtained,

$$
\mathcal{J}:= \begin{cases}\dot{x}(t) & =\mathcal{A}(\mu(t)) x(t)+B_{w}(\mu(t)) w(t), \\ y(t) & =C(\mu(t)) x(t),\end{cases}
$$

with $\mathcal{A}(\mu):=A(\mu)+B_{u}(\mu) K(\mu)$. 
Assume $\mathcal{A}(\mu)$ is stable for any pair $(\mu, \dot{\mu}) \in \Lambda \times \Omega$, then there is a parameter-dependent positive-definite matrix $P(\mu)=P(\mu)^{T}>0$ satisfying the following inequality

$$
\dot{P}(\mu)+\mathcal{A}(\mu) P(\mu)+P(\mu) \mathcal{A}(\mu)^{T}+B_{w}(\mu) B_{w}(\mu)^{\prime}<0 .
$$

In other words, suppose there is a continuously differentiable parameter-dependent matrix $\bar{P}(\mu)=\bar{P}(\mu)^{\prime}>$ 0 that solves the parameter-dependent controllability Gramian of the LPV system,

$$
\dot{\bar{P}}(\mu)+\mathcal{A}(\mu) \bar{P}(\mu)+\bar{P}(\mu) \mathcal{A}(\mu)^{T}+B_{w}(\mu) B_{w}(\mu)^{\prime}=0 .
$$

Therefore, there is a parameter-dependent matrix $M(\mu)=M(\mu)^{\prime}>0$ such that

$$
\dot{P}(\mu)+\mathcal{A}(\mu) P(\mu)+P(\mu) \mathcal{A}(\mu)^{T}+B_{w}(\mu) B_{w}(\mu)^{\prime}+M(\mu)=0,
$$

that implies $P(\mu)>\bar{P}(\mu)$. In other words, $P(\mu)$ is a parameter-dependent covariance matrix and the control covariance can be defined,

$$
U_{k}=\Phi_{k} K(\mu) P(\mu) K(\mu)^{T} \Phi_{k}^{T}<\bar{U}_{k}, \quad k=1,2, \cdots, n_{u} .
$$

where $\Phi_{k}$ is the $k$-th row of an identity matrix of size $n_{u} \times n_{u}$ associated with the $k$-th control channel.

Hence, the controller should exponentially stabilizes the closed-loop system and minimizes the upper bound of the ICC cost defined by,

$$
J_{I C C}=\operatorname{trace}\left(C(\mu) \bar{P}(\mu) C(\mu)^{T}\right) .
$$

Since the cost is parameter-dependent also, we introduce additional semi-definite (parameter-independent) matrix $W$ such that

$$
W-C(\mu) P(\mu) C(\mu)^{T}>0 .
$$

Therefore, minimizing the $\operatorname{trace}(W)$ corresponding to minimizing the upper bound of the ICC cost,

$$
\bar{J}_{I C C}=\operatorname{trace}(W) .
$$

More specifically, the goal is to find a gain-scheduling state-feedback controller to minimize (16) subject to the constraints (13).

Theorem 1. Given input covariance constraints $\bar{U}_{k}$ for $k=$ $1,2, \cdots, n_{u}$, and a sufficiently small scalar $\epsilon>0$. If there exist continuously differentiable parameter-dependent matrix $0<P(\mu)=P(\mu)^{T} \in \mathbb{R}^{n \times n}, G(\mu) \in \mathbb{R}^{n \times n}, Z(\mu) \in \mathbb{R}^{n_{u} \times n}$, and parameter-independent matrix $W=W^{T} \in \mathbb{R}^{n_{y} \times n_{y}}$ that solve the following optimization problem

$$
\min \operatorname{trace}(W) \text { s.t. }
$$

$$
\left[\begin{array}{ccc}
\psi(\mu)+\psi(\mu)^{T}-\dot{P}(\mu) & \star & \star \\
P(\mu)-G(\mu)+\epsilon \psi(\mu)^{T} & -\epsilon\left(G(\mu)+G(\mu)^{T}\right) & \star \\
B_{w}(\mu)^{T} & \boldsymbol{O}_{n_{w} \times n} & -I_{n_{w}}
\end{array}\right]<0,
$$

$$
\begin{array}{cc} 
& {\left[\begin{array}{cc}
W & C(\mu) G(\mu) \\
\star & G(\mu)+G(\mu)^{T}-P(\mu)
\end{array}\right]>0,} \\
{\left[\begin{array}{cc}
\bar{U}_{k} & \Phi_{k} Z(\mu) \\
\star & G(\mu)+G(\mu)^{T}-P(\mu)
\end{array}\right]>0, \quad k=1,2, \ldots, n_{u},}
\end{array}
$$

then the gain-scheduling controller

$$
K(\mu)=Z(\mu) G(\mu)^{-1},
$$

stabilizes the closed-loop system for any $(\mu, \dot{\mu}) \in \Lambda \times \Omega$, with the ICC cost bounded by

$$
\begin{aligned}
& \bar{J}_{\text {ICC }}=\operatorname{trace}(W)> \\
& \quad \operatorname{trace}\left(C(\mu) P(\mu) C(\mu)^{T}\right) \\
& \quad>\operatorname{trace}\left(C(\mu) \bar{P}(\mu) C(\mu)^{T}\right)=J_{\text {ICC }} .
\end{aligned}
$$

where $\Phi_{k}$ is (suitably chosen) input channel selection matrix and $\psi:=A(\mu) G(\mu)+B(\mu) Z(\mu)$.

Proof. Multiplying (17) by $\mathcal{T}(\mu)$ from left and by $\mathcal{T}(\mu)^{\prime}$ from right with

$$
\mathcal{T}(\mu)=\left[\begin{array}{ccc}
I & A(\mu) & \mathbf{0} \\
\mathbf{0} & \mathbf{0} & I
\end{array}\right],
$$

which leads to

$$
\left[\begin{array}{cc}
\mathcal{A}(\mu) P(\mu)+P(\mu) \mathcal{A}(\mu)-\dot{P}(\mu) & B_{w}(\mu) Q^{1 / 2} \\
Q^{1 / 2} B_{w}(\mu)^{T} & -I_{n_{w}}
\end{array}\right]<0,
$$

where $Q$ is a weighting matrix and $\mathcal{A}(\mu):=A(\mu) P(\mu)+$ $B_{u}(\mu) Z(\mu)$.

Taking the Schur complement of (23) yields

$$
-\dot{P}(\mu)+\mathcal{A}(\mu) P(\mu)+P(\mu) \mathcal{A}(\mu)+B_{w}(\mu) Q B_{w}(\mu)^{T}<0
$$

Consider now inequality (18), block $(2,2)$ dictates $G(\mu)+G(\mu)^{T}>P(\mu)>0$. Since the inequality is positive-definite and using the fact that $G(\mu)^{T} P(\mu)^{-1} G(\mu)>$ $G(\mu)+G(\mu)^{T}-P(\mu)$, the $(2,2)$ block can be replaced by $G(\mu)^{T} P(\mu)^{-1} G(\mu)$,

$$
\left[\begin{array}{cc}
W & C(\mu) G(\mu) \\
\star & G(\mu)^{T} P(\mu)^{-1} G(\mu)
\end{array}\right]>0 .
$$

Then, taking the Schur complement of (18) yields

$$
W-C(\mu) G(\mu)\left(G(\mu)^{-1} P(\mu) G(\mu)^{-T}\right) G(\mu)^{T} C(\mu)^{T}>0,
$$


that gives

$$
W>C(\mu) P(\mu) C(\mu)^{T} .
$$

Since $C(\mu) P(\mu) C(\mu)^{T}>C(\mu) \bar{P}(\mu) C(\mu)^{T}$,

$$
\begin{aligned}
\bar{J}_{I C C}=\operatorname{trace}(W)> & \operatorname{trace}\left(C(\mu) P(\mu) C(\mu)^{T}\right)> \\
\operatorname{trace}\left(C(\mu) \bar{P}(\mu) C(\mu)^{T}\right) & =J_{I C C .} .
\end{aligned}
$$

Therefore, considering (25), minimizing trace $(W) \mathrm{im}$ plies minimizing the upper bound of the ICC cost (26).

Following similar steps, the PLMIs (19) can be written as $\bar{U}_{k}>\Phi_{k} K(\mu) P(\mu) K(\mu)^{T} \Phi_{k}^{T}$ for $k=1,2, \cdots, n_{u}$, that implies the control covariance bounded by $\bar{U}_{k}$.

Some remarks are due for Theorem 1 in sequential order.

Remark 2. As a direct result of Theorem 1, robust ICC controller can be designed as special case of the LPV controller (20). More specifically, in the case where the scheduling parameter is not available for feedback in real-time, robust ICC controller can be designed. Simply, choosing the synthesis variables $(G(\mu)$ and $Z(\mu)$ ) to be parameter-independent ( $G$ and $Z$ ) as given in the following corollary.

Corollary 3. Given input covariance constraints $\bar{U}_{k}$ for $k=$ $1,2, \cdots, n_{u}$, and a sufficiently small scalar $\epsilon>0$. If there exist continuously differentiable parameter-dependent matrix $0<P(\mu)=P(\mu)^{T} \in \mathbb{R}^{n \times n}$, parameter-independent matrices $G \in \mathbb{R}^{n \times n}, Z \in \mathbb{R}^{n_{u} \times n}$, and $W=W^{T} \in \mathbb{R}^{n_{y} \times n_{y}}$ that solve the following optimization problem

$$
\text { min } \operatorname{trace}(W) \text { s.t. }
$$

$$
\begin{gathered}
{\left[\begin{array}{ccc}
\psi_{r}(\mu)+\psi_{r}(\mu)^{T}-\dot{P}(\mu) & \star & \star \\
P(\mu)-G+\epsilon \psi(\mu)^{T} & -\epsilon\left(G+G^{T}\right) & \star \\
B_{w}(\mu)^{T} & \boldsymbol{O}_{n_{w} \times n} & -I_{n_{w}}
\end{array}\right]<0,} \\
{\left[\begin{array}{cc}
W & C(\mu) G \\
\star & G+G^{T}-P(\mu)
\end{array}\right]>0,} \\
{\left[\begin{array}{cc}
\bar{U}_{k} & \Phi_{k} Z \\
\star & G+G^{T}-P(\mu)
\end{array}\right]>0, \quad k=1,2, \ldots, n_{u},}
\end{gathered}
$$

then the robust ICC controller

$$
K=Z G^{-1},
$$

stabilizes the closed-loop system with guaranteed ICC cost bounded by

$$
\begin{aligned}
\bar{J}_{I C C} & =\operatorname{trace}(W)> \\
& \operatorname{trace}\left(C(\mu) P(\mu) C(\mu)^{T}\right) \\
& >\operatorname{trace}\left(C(\mu) \bar{P}(\mu) C(\mu)^{T}\right)=J_{I C C} .
\end{aligned}
$$

where $\Phi_{k}$ is (suitably chosen) input channel selection matrix and $\psi_{r}:=A(\mu) G+B(\mu) Z$.
Remark 4. The synthesis conditions of Theorem 1 are parametrized with $\mu(t)$ (polytopic vertices) instead of $\theta(t)$ since a simple change of variables can be used to convert the original LPV model into the polytopic domain.

Remark 5. The conditions of Theorem 1 are expressed as Parametrized Linear Matrix Inequalities (PLMIs). Hence, PLMIs can be interpreted as special type of convex optimization with infinite constraints. Therefore, relaxation methods need to be applied in order to solve this optimization problem. In this work, matrix coefficients check relaxation approach using Pólya's theorem [16] is utilized to obtain the optimal LPV controller.

Remark 6. The scalar $\epsilon$ in (17) can be fixed as a sufficiently small constant beforehand. This constant can be viewed as additional degree of freedom to avoid numerical problems and improve the achieved performance.

Remark 7. The software package ROLMIP [?] is used in this work to perform PLMI relaxation. More specifically, it is used to convert the infinite dimensional LMIs into finite set LMIs. SeDuMi [?] and YALMIP [?] are also used with ROLMIP to obtain the optimal solution.

\section{Numerical Examples}

\subsection{Example: 1}

Consider the following LPV system [? ]:

$$
\begin{gathered}
A(\theta(t))=\left[\begin{array}{cc}
25.9-60 \theta(t) & 1 \\
20-40 \theta(t) & 34-64 \theta(t)
\end{array}\right], \quad B_{u}=\left[\begin{array}{l}
3 \\
2
\end{array}\right], \\
B_{w}=\left[\begin{array}{l}
-0.03 \\
-0.47
\end{array}\right], \quad C=\left[\begin{array}{ll}
1 & 0
\end{array}\right] .
\end{gathered}
$$

with $\theta(t) \in[0,1]$ and $\dot{\theta}(t) \in[-1,1]$. First of all the system has been converted from the actual parameter domain into polytopic domain. More specifically, from $\theta$-domain into $\mu$-domain, where

$$
\mu_{1}(t)=\frac{\theta(t)-\underline{\theta}}{\bar{\theta}-\underline{\theta}}, \quad \mu_{2}(t)=1-\mu_{1}(t),
$$

with $\underline{\theta}=-1$ and $\bar{\theta}=1$.

Then, Theorem 1 is used to design LPV controllers with various constraints on the control input. Table 1 shows the number of LMI (optimization) variables and LMI constraints for Theorem 1 associated with Example 1.

Three cases have been studied with input constraints, $\bar{U}=9.5,10$ and 10.5 , respectively. For the first case, 
Table 1: Number of LMI variables and constraints for Theorem 1 (Example 1).

\begin{tabular}{c|c|c}
\hline & \# of LMI variables & \# of LMI rows \\
\hline Theorem 1 & 19 & 46 \\
\hline
\end{tabular}

Table 2: ICC cost and Control covariance at the system vertices for Example $1\left(\epsilon=10^{-4}\right)$.

\begin{tabular}{|c|c|c|c|}
\hline \multicolumn{4}{|c|}{$\bar{U}=9.5$} \\
\hline & vertex\#1 & vertex\#2 & $\operatorname{trace}(W)$ \\
\hline$U$ & 9.4994 & 9.4873 & 0.0586 \\
\hline$\overline{J_{I C C}}$ & 0.0585 & 0.0542 & \\
\hline$\overline{\bar{J}}_{I C C}$ & 0.0586 & 0.0585 & \\
\hline$K$ & {$\left[\begin{array}{ll}-6.645 & -63.165]\end{array}\right.$} & {$[12.333-46.966]$} & \\
\hline \multicolumn{4}{|c|}{$\overline{\bar{U}=10}$} \\
\hline & vertex\#1 & vertex\#2 & $\operatorname{trace}(W)$ \\
\hline$U$ & 9.9991 & 9.9874 & 0.0578 \\
\hline$J_{I C C}$ & 0.0578 & 0.0559 & \\
\hline$\overline{\bar{J}}_{I C C}$ & 0.0578 & 0.0578 & \\
\hline$K$ & {$\left[\begin{array}{ll}-6.614 & -75.834\end{array}\right]$} & {$\left[\begin{array}{ll}12.464 & -56.077\end{array}\right]$} & \\
\hline \multicolumn{4}{|c|}{$\overline{\bar{U}}=10.5$} \\
\hline & vertex\#1 & vertex\#2 & $\operatorname{trace}(W)$ \\
\hline$U$ & 10.4987 & 10.4861 & 0.0575 \\
\hline$J_{I C C}$ & 0.0574 & 0.0563 & \\
\hline$\overline{\bar{J}}_{I C C}$ & 0.0575 & 0.0574 & \\
\hline K & {$\left[\begin{array}{ll}-6.704 & -86.821]\end{array}\right.$} & {$[12.534-65.554]$} & \\
\hline
\end{tabular}

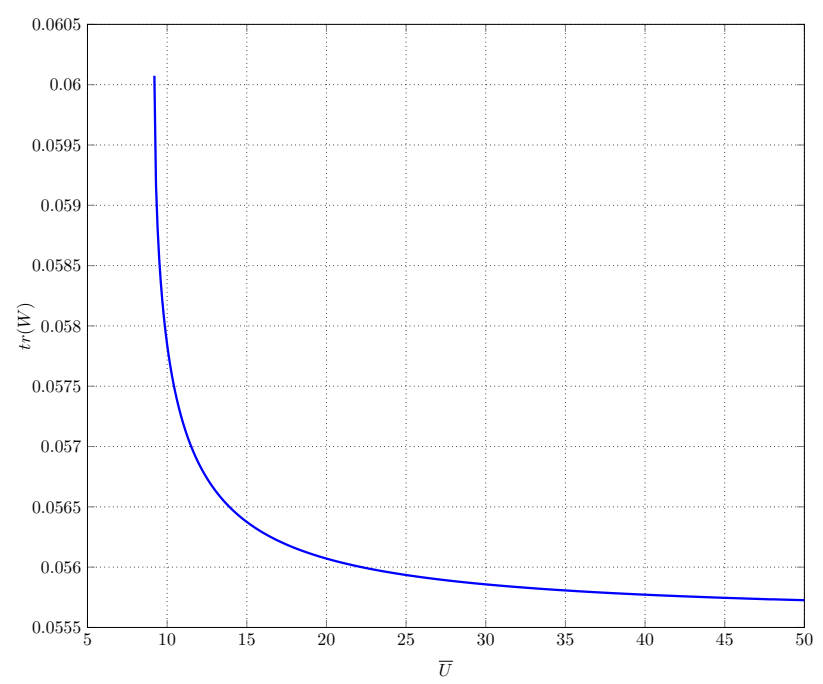

Figure 1: The upper bound on the output performance versus control constraints.

$\bar{U}=9.5$ is used in Theorem 1 to design the optimal LPV controller satisfying this control constraints. Table 2 illustrates the design results at the vertices of the polytope with
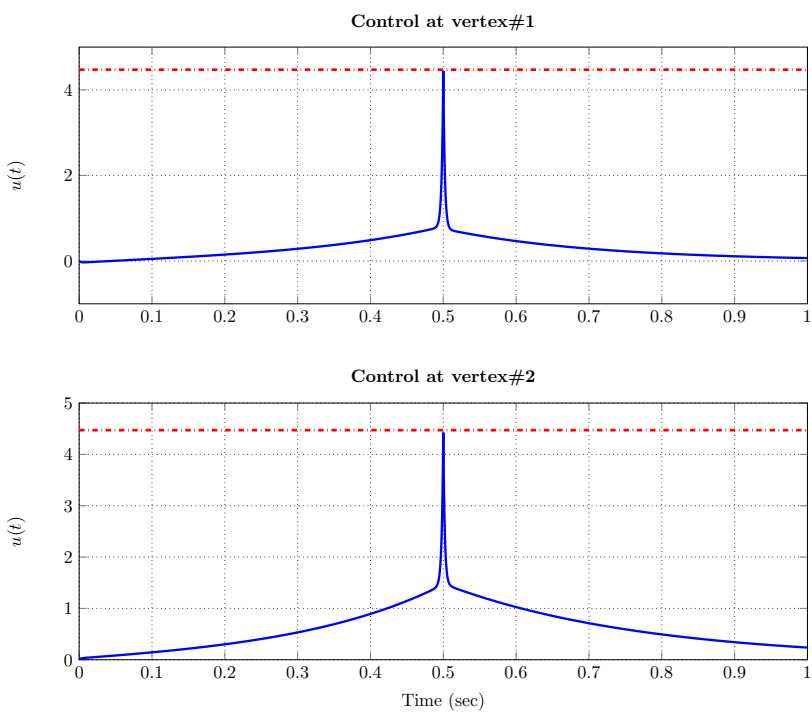

Figure 2: Worst case control response at vertex\#1 and vertex\#2 (blue) and the $\sqrt{\bar{U}}=\sqrt{20}$ in the dash-dotted (red).

the vertex controller,

$$
K_{1}=\left[\begin{array}{ll}
-6.645 & -63.165
\end{array}\right], \quad K_{2}=\left[\begin{array}{lll}
12.333 & -46.966
\end{array}\right] .
$$

It is clear from Table 2 that the utilized control energy at the two vertices are 9.4994 and 9.4873 which satisfy the constraint $\bar{U}=9.5$. With this constraint, the optimal ICC costs are 0.0585 and 0.0542 at the vertices. Note that $\operatorname{trace}(W)=0.0586$ is the upper bound for the ICC cost. This bound is very tight demonstrating optimality of the design.

For the case $\bar{U}=10$, the utilized control energy at the vertices are 9.9991 and 9.9874 with upper bound of the ICC cost is 0.0578 . Similarly, for the case $\bar{U}=10.5$, the utilized control energy at the vertices are 10.4987 and 10.4861 with upper bound of the ICC cost is 0.0575 .

Figure 1 illustrates the relationship between the optimized output performance versus the control constraints. As expected, the output performance is getting better as the constraints on the control relaxed and vise versa.

The worst case control response for $\bar{U}=20$ is shown in Fig. 2 for both vertices demonstrating the control input constraint is totally respected. This response illustrating that the control resources are optimally utilized, neither commanding the actuator higher than the specified constraint nor providing low actuation signal. 
Table 3: ICC cost and Control covariance at the system vertices for Example $2(\epsilon=0.05)$.

\begin{tabular}{|c|c|c|c|c|c|}
\hline \multicolumn{5}{|c|}{$\bar{U}=0.5$} & \multirow[b]{2}{*}{$\operatorname{trace}(W)$} \\
\hline & \multicolumn{2}{|r|}{ vertex\#1 } & & vertex\#2 & \\
\hline$U$ & \multicolumn{2}{|r|}{0.4847} & & 0.4985 & 0.0321 \\
\hline$\overline{J_{I C C}}$ & \multicolumn{2}{|r|}{0.0282} & & 0.0305 & \\
\hline$\overline{\bar{J}}$ ICC & \multicolumn{2}{|r|}{0.0316} & & 0.0316 & \\
\hline \multirow[t]{3}{*}{$K$} & {$[-0.782$} & $-7.615-1.736]$ & {$[-0.783$} & $-8.664-0.998]$ & \\
\hline & \multicolumn{4}{|c|}{$\bar{U}=2$} & \\
\hline & \multicolumn{2}{|r|}{ vertex\#1 } & & vertex\#2 & $\operatorname{trace}(W)$ \\
\hline$U$ & \multicolumn{2}{|r|}{1.9740} & & 1.9810 & 0.0134 \\
\hline$J_{I C C}$ & \multicolumn{2}{|r|}{0.0112} & & 0.0115 & \\
\hline$\overline{\bar{J}}$ ICC & \multicolumn{2}{|r|}{0.0130} & & 0.0130 & \\
\hline \multirow[t]{3}{*}{$K$} & {$[-12.757$} & $-28.987-5.023]$ & {$[-12.822$} & $-30.044-4.834]$ & \\
\hline & \multicolumn{4}{|c|}{$\bar{U}=4$} & \\
\hline & \multicolumn{3}{|c|}{ vertex\#1 } & vertex\#2 & $\operatorname{trace}(W)$ \\
\hline$U$ & \multicolumn{3}{|c|}{3.9419} & 3.9588 & 0.0101 \\
\hline$J_{I C C}$ & \multicolumn{3}{|c|}{0.0078} & 0.0079 & \\
\hline$\overline{\bar{J}_{I C C}}$ & \multicolumn{2}{|r|}{0.0097} & & 0.0097 & \\
\hline$K$ & {$[-25.224$} & $-47.757-9.139]$ & {$[-25.494$} & $-48.957-9.034]$ & \\
\hline
\end{tabular}

\subsection{Example: 2}

Consider the modified version of the system presented in [11]:

$$
\begin{gathered}
A(\theta(t))=\left[\begin{array}{ccc}
0 & 1 & 0 \\
-1 & 0.1 \theta(t) & 1 \\
0 & 0 & -10
\end{array}\right], \quad B_{u}=\left[\begin{array}{l}
0 \\
0 \\
1
\end{array}\right], \\
B_{w}=\left[\begin{array}{l}
0 \\
0 \\
1
\end{array}\right], \quad C=\left[\begin{array}{ccc}
1 & 0.5 & 0 \\
0 & 0 & 0.5 \\
1 & 1 & 0
\end{array}\right] .
\end{gathered}
$$

with $\theta(t) \in[0,1]$ and $\dot{\theta}(t) \in[-1,1]$.

After converting the system into polytopic domain, Theorem 1 is used to synthesize LPV controller with ICC constraints. Table 4 shows the number of optimization variables and LMI constraints associated with Theorem 1 for this example. Similarly, three cases have been studied with constraints $\bar{U}=0.5,2$, and 4 . Table 3 presents the design results in terms of vertex controllers and the achieved output performance for each individual case. It is clear to see the control energy satisfying the specified constraints. Figure 3 demonstrates the relationship between the control constraints and the achieved $J_{\text {ICC }}$.
Table 4: Number of LMI variables and number of LMI rows for Theorem 1 (Example 2).

\begin{tabular}{l|c|c}
\hline & \# of LMI variables & \# of LMI rows \\
\hline Theorem 1 & 42 & 68 \\
\hline
\end{tabular}

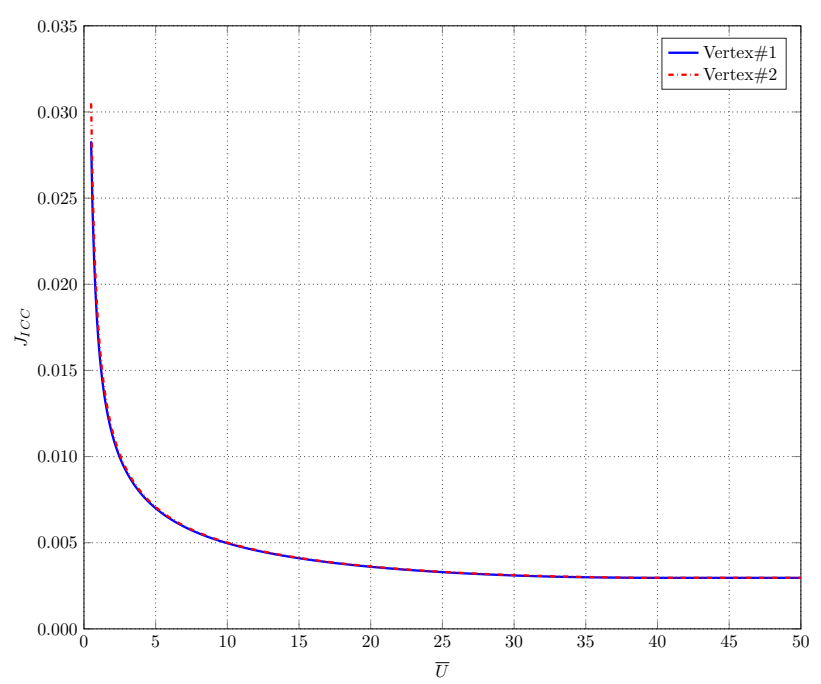

Figure 3: The ICC cost versus control constraints at vertex \#1 and vertex \#2. 


\section{Conclusions}

New synthesis conditions have been developed to design state-feedback LPV controller satisfying constraints on control covariance. These conditions are given in terms of Parameterized Linear Matrix Inequalities (PLMIs). Two numerical examples is presented to illustrate the benefit of the developed approach.

\section{References}

[1] Apkarian, Pierre, and Richard J. Adams. Advanced gainscheduling techniques for uncertain systems. Advances in linear matrix inequality methods in control, Society for Industrial and Applied Mathematics, 2000. 209-228.

[2] Rugh, Wilson J., and Jeff S. Shamma. Research on gain scheduling. Automatica 36.10 (2000): 1401-1425.

[3] Hoffmann, Christian, and Herbert Werner. "A survey of linear parameter-varying control applications validated by experiments or high-fidelity simulations." IEEE Transactions on Control Systems Technology 23.2 (2015): 416-433.

[4] Shamma, Jeff S., and Michael Athans. "Gain scheduling: Potential hazards and possible remedies." IEEE Control Systems Magazine 12.3 (1992): 101-107.

[5] Leith, Douglas J., and William E. Leithead. "Survey of gainscheduling analysis and design." International journal of control 73.11 (2000): 1001-1025.

[6] Al-Jiboory, Ali Khudhair, Guoming G. Zhu, and Jongeun Choi. "Guaranteed performance state-feedback gain-scheduling control with uncertain scheduling parameters." Journal of Dynamic Systems, Measurement, and Control 138.1 (2016): 014502.

[7] Al-Jiboory, Ali Khudhair, and Guoming G. Zhu. "Improved synthesis conditions for mixed $\mathrm{H}$ 2/Hấđđ gain-scheduling control subject to uncertain scheduling parameters." International Journal of Control 90.3 (2017): 580-598.

[8] Al-jiboory, Ali Khudhair, et al. LPV modeling of a flexible wing aircraft using modal alignment and adaptive gridding methods. Aerospace science and technology 66 (2017): 92-102.
[9] Al-jiboory, Ali Khudhair, et al. Linear matrix inequalities approach to input covariance constraint control with application to electronic throttle. Journal of Dynamic Systems, Measurement, and Control 137.9 (2015): 091010.

[10] Al-Jiboory, Ali Khudhair, Guoming Zhu, and Cornel Sultan. LMI control design with input covariance constraint for a tensegrity simplex structure. ASME 2014 Dynamic Systems and Control Conference. American Society of Mechanical Engineers, 2014.

[11] Zhu, Guoming, M. A. Rotea, and R. Skelton. A convergent algorithm for the output covariance constraint control problem. SIAM Journal on Control and Optimization 35.1 (1997): 341-361.

[12] White, Andrew, Guoming Zhu, and Jongeun Choi. A linear matrix inequality solution to the input covariance constraint control problem. ASME 2013 Dynamic Systems and Control Conference. American Society of Mechanical Engineers, 2013.

[13] Al-Jiboory, Ali Khudhair, Guoming Zhu, and Cornel Sultan. LMI control design with input covariance constraint for a tensegrity simplex structure. ASME 2014 Dynamic Systems and Control Conference. American Society of Mechanical Engineers, 2014.

[14] Al-Jiboory, Ali Khudhair, et al. Linear matrix inequalities approach to input covariance constraint control with application to electronic throttle. Journal of Dynamic Systems, Measurement, and Control 137.9 (2015): 091010.

[15] Hsieh, C., R. E. Skelton, and F. M. Damra. Minimum energy controllers with inequality constraints on output variances. Optimal Control Applications and Methods 10.4 (1989): 347-366.

[16] Oliveira, Ricardo CLF, and Pedro LD Peres. Parameterdependent LMIs in robust analysis: Characterization of homogeneous polynomially parameter-dependent solutions via LMI relaxations. IEEE Transactions on Automatic Control 52.7 (2007): 1334-1340.

[17] Geromel, JosÃl' C., and Patrizio Colaneri. Robust stability of time varying polytopic systems. Systems \& Control Letters 55.1 (2006): 81-85.

[18] Chesi, Graziano, et al. Robust stability of time-varying polytopic systems via parameter-dependent homogeneous Lyapunov functions. Automatica 43.2 (2007): 309-316. 